\title{
Inclusión del enfoque de género en la enseñanza de las ciencias mediante el estudio de biografías de mujeres científicas
}

\author{
Carmen Solís-Espallargas ${ }^{1, a}$ \\ ${ }^{1}$ Departamento de Didáctica de las Ciencias Experimentales y Sociales. Universidad de Sevilla. Sevilla. \\ España. \\ acarmensolise@us.es,
}

[Recibido: 20 Noviembre 2017. Revisado: 31 Enero 2018. Aceptado: 31 Marzo 2018]

\begin{abstract}
Resumen: En este trabajo se sugiere, en primer lugar, la necesidad de que Maestras y Maestros de Primaria en formación construyan un conocimiento general de la ciencia relacionado tanto con los contenidos más estructurantes de las disciplinas implicadas como con la Historia de la ciencia desde un enfoque de género, que les permita analizar críticamente las relaciones entre ciencia-tecnología y género-sociedad a lo largo de la historia y en el presente. En segundo lugar se presenta una investigación cualitativa a partir de una propuesta didáctica realizada por estudiantes de Didáctica de las Ciencias en el Grado de Educación Primaria de la Universidad de Granada sobre biografías de mujeres científicas. Finalmente se discuten los resultados de la investigación con el objeto de concluir con claves que nos orienten en el diseño de propuestas formativas para la enseñanza de las ciencias desde un enfoque de género.
\end{abstract}

Palabras clave: Enseñanza de las ciencias; Biografías; Género; Historia de la ciencia; Educación primaria.

Inclusion of gender approach in teaching of sciences with biographies of female scientists

\begin{abstract}
This paper is aimed, firstly, at suggesting the need for in-training Primary Education Teachers to build up general knowledge about science structuring contents as well as history of science from a gender perspective. This training in science should help students to analyse the relationships between science-technology and gendersociety throughout history and at present. Secondly, a qualitative research carried out about a proposal developed by Science Didactics students of the Degree in Primary Education in the University of Granada about biographies of female scientists is described. Finally, the results of the research are presented, all leading to some key ideas on how to introduce the gender approach in the teaching of science.
\end{abstract}

Keywords: Teaching of sciences, Biographies, Gender, History of science, Primary education.

Para citar este artículo: Solís-Espallargas, C. (2018) Inclusión del enfoque de género en la enseñanza de las ciencias mediante el estudio de biografías de mujeres científicas. Revista Eureka sobre Enseñanza y Divulgación de las Ciencias 15(3), 3602. doi: 10.25267/Rev_Eureka_ensen_divulg_cienc.2018.v15.i3.3602

\section{Introducción}

La Ciencia se encuentra presente en casi todas nuestras acciones, hábitos y tareas. El conocimiento científico debe ser en la actualidad parte del saber esencial de las personas para que puedan entender la realidad con racionalidad y libertad y construyan opiniones libres dotadas de argumentos para tomar decisiones. Es por ello, que la educación científica resulta fundamental como parte de la educación de las personas (Marco-Stiefel 2004, Acevedo 2004).

Por ello, las y los docentes necesitan estar muy bien preparados en una materia fundamental como es el conocimiento científico y la naturaleza de la ciencia, para saber enseñar a manejar tanta información, a discernir la ciencia de lo que no lo es y a tomar decisiones de vida justificadas científicamente. La importancia de la enseñanza de la ciencia básica en los

\author{
Revista Eureka sobre Enseñanza y Divulgación de las Ciencias \\ Universidad de Cádir. APAC-Eureka. ISSN: 1697-011X \\ bttp:/ / dx.doi.org/10.25267/Rev_Eureka_ensen_divulg_cienc.2018.v15.i3.3602 \\ http:/ / reuredc.uca.es
}


primeros niveles educativos de Educación Infantil y Primaria, es muy defendida por autores tales como Eshach y Fried (2005); Muñoz et al. (2012); Pedrinaci et al. (2013); Rivero et al. (2017); entre otros.

Para una buena educación en ciencias, los maestros y maestras de Primaria en formación no sólo necesitan construir un conocimiento general de la ciencia relacionados con los contenidos más estructurantes de las disciplinas implicadas; sino también sobre la historia de la ciencia, es decir, la naturaleza epistemológica de este conocimiento, las estrategias con la que se construye y las relaciones entre ciencia-tecnología y género-sociedad a lo largo de la historia y en el presente; desde un enfoque complejo, integrador y no neutral en el que resulta fundamental enfatizar, por su carencia, el enfoque de género.

Hablar de género en la enseñanza de las ciencias plantea en los maestros y maestras en formación cuestiones políticas y epistemológicas sobre cómo y en que contextos se ha hecho y se hace ciencia. Significa comenzar a indagar sobre la ideología cultural que subyace tras la ciencia y la tecnología que contribuye al control político y se manifiesta en la cultura popular. Significa tomar conciencia tanto de la exclusión que han tenido y continúan teniendo las mujeres como sujeto en ciencia así como objetos de la misma no siendo extrapolables los resultados obtenidos con muestras masculinas a lo femenino (García y Pérez 2017). Significa cuestionar la visión cosmológica de la ciencia en el contexto masculino y analizar cómo es la naturaleza del conocimiento científico y cómo se ha desarrollado y se desarrolla la epistemología de la ciencia.

Significa superar las pretensiones positivistas de neutralidad y objetividad de la ciencia, libre de influencias, valores sociales y emociones y abrir las puertas a las críticas psicosociales de la ciencia, entre otras, las críticas feministas. Significa poner en cuestión valores dictados por los objetivos de la ciencia tales como la adecuación empírica, simplicidad, consistencia, novedad y estabilidad frente a otros valores tales como heterogeneidad ontológica, interacción mutua, aplicabilidad a las necesidades humanas y difusión del poder (Manassero y Vázquez 2003). Significa analizar cómo es la epistemología de la ciencia que se enseña en las aulas de Educación Primaria y su influencia tanto en la concepción de ciencia que se aprende así como, el género se convierte en una variable que condiciona la elección profesional futura. Hablar de género en la enseñanza de la ciencia significa comenzar por el análisis del proceso de socialización que han tenido los docentes en formación y cómo en su proceso personal se han interiorizado los estereotipos de género y la influencia que pueden tener sus prácticas de enseñanza en el aula. Significa demandar la formación necesaria en las facultades de Educación tanto para el profesorado universitario como para el estudiantado con el fin de superar la brecha de género existente en la enseñanza de las ciencias y la inclusión de este enfoque en los diseños curriculares. En una época en la que estamos viviendo la aplicación de reformas del sistema educativo, no sólo en España sino en muchos otros países ( $\mathrm{y}$ especialmente en países latinoamericanos) significa situar el enfoque de género como uno de los elementos claves de la construcción educativa.

Cualquier intento de renovar la realidad educativa ha de partir de una reflexión, en profundidad, acerca del tipo de escuela que se propone. Resultan necesarios planteamientos como: ¿qué tipo de conocimiento creemos que hay que proporcionar hoy a los jóvenes sobre ciencia?; y desde un plano más global: ¿qué cultura escolar habría que proponerles?; ¿qué tipo de socialización superaría la brecha de género?; ¿qué vinculaciones existen entre el modelo de cultura escolar y los sistemas de ideas -no tan formalizados, pero funcionales- que el alumnado maneja al margen de la enseñanza escolar?

Aunque estas son cuestiones de gran calado que merece especial abordaje, en este trabajo queremos matizar que cuando se plantea la necesidad de renovación no nos referimos tanto al 
sistema legalmente vigente, sistema que en el contexto español recoge como uno de sus fines de la enseñanza de Educación Primaria la igualdad de género (art. 3 Decreto 97/2015); sino al sistema realmente operante, es decir, el que se lleva a cabo realmente en los centros escolares. Por ejemplo, cómo es el sistema de organización del centro (si se reproducen roles de género), cómo es la dinámica habitual de la enseñanza en las aulas (si se incorpora el enfoque de género), si se tienen en cuenta las carencias formativas de los docentes para incluir la perspectiva de género en sus propuestas didácticas o qué tipos de libros de texto se utilizan y qué contenidos recogen, etc., sobre todo; en la concepción social dominante acerca del papel que ha de jugar la enseñanza en la superación de la brecha de género, concepción que suele resultar ser más conservadora en su práctica que en relación al propio sistema legal vigente.

\section{Marco teórico}

La comprensión de la ciencia va más allá del saber enciclopédico sobre los principales hechos, conceptos y principios, como ha defendido la enseñanza tradicional (Vázquez, Acevedo, Manassero y Acevedo 2001). En el marco de una alfabetización científica el estudio sobre la naturaleza de la ciencia, la historia y la filosofía de la ciencia ha ido cobrando protagonismo en los programas de formación docente con el objetivo de ofrecer una formación epistemológica, histórica e ideológica sobre la ciencia.

La Historia de la ciencia aporta una comprensión más profunda y no sólo desmonta la imagen de la Ciencia como un bloque acabado e inalterable sino que, el enfoque histórico permite poner en discusión la dimensión humana de la Ciencia (García Díaz 1995). A menudo se entiende la ciencia como una actividad neutra, no influenciada por la sociedad en la que se desarrolla, ni por intereses particulares, y cuyo máximo objetivo es la búsqueda de "la verdad". Sin embargo, existen ejemplos que ponen de manifiesto que los criterios que guían la investigación están sesgados por estereotipos o arquetipos sociales (Rivero y Aguado 2011).

El estudio sobre la historia de la ciencia permite abordar este enfoque de la neutralidad de la ciencia y de los límites de su independencia hacia lo social, ya que la ciencia no ha permanecido al margen del dominio entre otros, del modelo patriarcal en el que se ha enmarcado a lo largo de la historia. Concretamente, permite hacer una reflexión y toma de conciencia sobre cómo se ha ido construyendo la Ciencia y desde una mirada crítica cómo se han excluido muchas aportaciones de saberes y visiones del mundo en los contenidos de enseñanza, principalmente de las mujeres en la ciencia (Barale y María 2006; Novo 2007; Vega et al. 2010).

Sin embargo, a pesar de la exclusión recurrente de los lugares legítimos de la ciencia, las mujeres desde siempre han producido conocimiento aunque carezca de visibilidad y se niegue socialmente. Autoras como Urteaga (2010) propone que si se desea hacer emerger el lugar de las mujeres en la producción del saber científico no hay que limitarse a los espacios más legítimos, a las instituciones oficiales y a las grandes revistas científicas, para pensar la intrincación y variedad de los mundos en los que se produce el saber, especialmente en aquellos espacios dominados históricamente por las mujeres como por ejemplo antiguamente la obstetricia o la botánica. En actuales estudios como los llevados a cabo por la Universidad Complutense de Madrid sobre el Fomento de la igualdad de género en la información científica en los medios de comunicación españoles (García Nieto 2013), se concluye que apenas un $12 \%$ de las menciones de referencias científicas digitales en Internet corresponden a mujeres investigadoras, frente al $88 \%$ de las citas dedicadas a los hombres investigadores, por lo que las mujeres continúan siendo prácticamente invisibles actualmente en los mensajes científicos publicados en Internet. Este estudio concluye con la irrepresentabilidad de las mujeres científicas españolas en las informaciones de ciencia, investigación y tecnología, 
publicadas en los medios de comunicación españoles, hecho que refuerza la tendencia histórica de exclusión.

Autoras como Magallón (2016) plantean que la importancia de la invisibilidad no sólo reside en la historia de la ciencia, sino también de la influencia en la epistemología de la ciencia. Esta autora propone que hay que revisar lo que se entiende por ciencia, ver si en la transmisión científica se han borrado actividades y conocimientos que no encajaban en el esquema de la corriente principal masculina y si la pretendida objetividad que proclama es tal o si, como empresa humana, lleva en sí misma huellas/sesgos de género (y otros) que ponen en cuestión el carácter universal que se auto atribuye. Harding (1991) sostiene incluso que las mujeres podrían hacer ciencia de una manera diferente y una ciencia diferente de la realizada hasta ahora, desde la formulación de los problemas hasta el análisis de resultados, pasando por la toma de datos.

Desde un enfoque epistemológico, la naturaleza del conocimiento científico históricamente se ajusta a una visión cosmológica masculina en la que predomina una concepción positivista de la ciencia, objetiva, racional, inductiva, neutra y analítica; proyectando una imagen que coincide con las características asignadas al estereotipo masculino y, en este sentido, se dice que la ciencia tiene una marca de género masculina, que se ve agravada por la circunstancia crucial de que esté dominada por los hombres (Manassero y Vázquez 2013). Estas influencias positivistas actualmente continúan activas en el seno de la comunidad científica (Vázquez et al. 2001) y por tanto, en los docentes y como consecuencia en el sistema educativo.

El profesorado permanece inmerso en el proceso de socialización, en el que su cosmovisión se encuentra influenciada por el modelo hegemónico que continúa siendo masculino. Esto tiene como consecuencia la interiorización de los estereotipos de género que este modelo establece y parece razonable que estos modelos puedan activarse y transmitirse a través de las prácticas de enseñanza en el aula. Como ejemplo de ello, en el estudio iniciado por la Universidad de la Laguna sobre Valores y sesgos en el conocimiento científico y su trasmisión (Gómez et al. 2008) se obtuvo como primeras conclusiones que en el profesorado de ciencias de esta universidad así como en el profesorado de los centros de secundaria aparecen sesgos de género respecto a las estudiantes en la enseñanza de la ciencia y las tecnologías. Estos sesgos están relacionados con las capacidades cognitivas, las destrezas, las actitudes y las interacciones de las estudiantes y los estudiantes y determinan que los consideren a ellos superiores y por tanto, a ellas inferiores en el aprendizaje de la ciencia y las tecnologías.

Los resultados de este trabajo muestran que esta tendencia es dominante y superior a la tendencia a creer que los estudiantes y las estudiantes son iguales entre ellos o que ellas son superiores. Aunque los datos obtenidos no pueden generalizarse estadísticamente las investigadoras e investigadores plantean que dadas las comunes condiciones sociales, culturales y estructurales compartidas por el profesorado de ciencias y tecnologías en todo el Estado español, arriesgan la hipótesis sobre la generalización de los resultados hallados. La conducta del profesorado en el aula y las posibles diferencias que hacen entre chicas y chicos contribuye a fomentar los estereotipos de género junto a otros factores escolares como la imagen masculina dominante de la ciencia que se refleja en el currículo de ciencias y en los libros de texto.

La ausencia de mujeres científicas de los libros de texto de ciencias y tecnología es un hecho claro y abrumador (Manassero y Vázquez 2013). Para estos autores, este aspecto resulta especialmente grave para el objetivo de la igualdad de sexos, propuesto explícitamente para la educación general, al compararse con situaciones anteriores cuando el principio de igualdad de sexos no estaba legalmente reconocido y se obtienen los mismos resultados en los nuevos libros de texto correspondientes a planes de estudio donde la educación para la igualdad de 
sexos está explícitamente reconocido y recomendado para todas las áreas. Hecho que se constata en el estudio de Álvarez y Soneira (1991) realizado con muestras de libros de texto impresos hace más de 10 o 15 años. Estudios más recientes (López-Navajas 2014) sobre la presencia de las mujeres en los manuales de la Enseñanza Secundaria Obligatoria (ESO) en España muestran una muy escasa presencia de mujeres -12,8\%-, el cual revela la continua falta de consideración social de las mujeres y su práctica exclusión de la visión de mundo que se traslada desde la enseñanza. Otro dato relevante de este estudio concluye en que la presencia de mujeres disminuye a medida que los cursos aumentan de nivel y los contenidos ganan en profundidad. En la segunda etapa $\left(3 .^{\circ}\right.$ y $4 .^{\circ}$ de ESO) su presencia es menor $(10 \%)$ que en la primera (1..$^{\circ}$ y $2 .^{\circ}$ de ESO), con un $13 \%$. La pérdida de importancia de las mujeres en los contenidos fundamentales es otro indicador de su falta de relevancia.

Además de suponer una considerable falta de rigor en los contenidos académicos perpetuando desigualdades, la falta de modelos científicos femeninos, sociales y personales en los libros de texto no solo no favorecen, sino que constituyen un elemento de exclusión activa para la identificación de las mujeres con la ciencia (Lomas 2002; Blanco García 2004; Espigado 2004; Rodríguez Martínez 2004; López y García-Molins 2014).

Una de las primeras propuestas de enseñanza de las ciencias con enfoque de género se inició en los años ochenta con el proyecto GIST (Girls into Science and Technology, Smail 1982), que pretendía obtener resultados que pudieran ayudar al profesorado de ciencias a considerar el género como una variable relevante a la hora de diseñar su currículo y la enseñanza y estimularlo a renovar sus prácticas de enseñanza en este sentido.

Esta propuesta puede ser una herramienta importante para hacer de la educación en ciencias una enseñanza más igualitaria que supere los estereotipos de género en la enseñanza de las ciencias. Sin embargo consideramos que para que el profesorado sea capaz de desarrollar estas competencias resulta fundamental dirigir las propuestas de acción en la formación inicial de docentes en ciencias.

Es por ello que en este trabajo ponemos de relieve la necesidad de promover en la formación inicial de maestras y maestros una capacitación científica más humana y social ligada al contexto real en el que se desenvuelve el alumnado. Este planteamiento nos permite abordar aspectos epistemológicos, históricos e ideológicos de la ciencia desde un enfoque complejo, integrador y no neutral. Hablar de género en la ciencia plantea en los maestros y maestras en formación cuestiones políticas y epistemológicas sobre cómo y en qué contextos se hace ciencia.

Aunque generalmente se cree que la ciencia es una construcción del saber colectiva e universal, la exclusión histórica y actual de las mujeres en los ámbitos legítimos de la ciencia descritas anteriormente, conlleva replantear a los docentes en formación sobre la producción del saber y los contextos donde se realiza.

Concretamente en el estudio sobre la Historia de la ciencia mediante la investigación biográfica permite una aproximación crítica sobre la epistemología de la ciencia a partir de la investigación sobre historias de vida de mujeres científicas más allá de los universos masculinos que constituyen los lugares de la ciencia oficial.

Desde fines del siglo XIX, las mujeres han accedido poco a poco al mismo saber que los hombres gracias a la voluntad prodigiosa de ciertas mujeres que quisieron y pudieron integrarse en estos universos masculinos. El estudio que se materializó en el libro Pioneras españolas en las ciencias (Magallón 2004) recoge que desde 1910 hasta 1936, el ritmo con el que crecían las mujeres que se encaminaban a carreras de ciencias fue mayor que el de aquellas que se dirigían al resto de las carreras. 
El género biográfico en ciencia puede ser una herramienta fundamental de análisis para promover una educación científica crítica que visibilice y empodere los valores femeninos como un aspecto esencial de la experiencia humana y proponga una nueva visión de la ciencia que pueda incorporar esos valores.

Hemos de tener en cuenta que en muchos de los análisis sobre biografías de mujeres científicas encajan en el molde de «historia de grandes hombres» sólo sustituyendo mujeres por hombres (Maffía 2007). El enfoque de estos estudios ubican los logros dentro del mundo masculino, demostrando que las mujeres han hecho contribuciones importantes a la corriente principal de la ciencia que de por sí es masculina, cuestión que tendremos presente en el análisis de los datos.

Desde este planteamiento, presentamos una investigación a partir de los trabajos que realizan maestros y maestras en formación sobre biografías de mujeres científicas cuyo análisis y discusión de resultados pretende darnos claves que nos orienten en el diseño de propuestas formativas para la enseñanza de las ciencias desde un enfoque de género.

\section{Metodología}

El objetivo concreto de esta investigación se basa en determinar qué elementos destacan los estudiantes del estudio de biografías de mujeres científicas y cuáles de esos elementos están directamente relacionados con el género.

Para ello nos planteamos las siguientes preguntas ¿Qué tipo de biografías escogen, es decir, de qué época y campo científico? ¿Por qué escogen esa biografía? ¿Qué elementos destacan de la biografía?

La investigación se realiza sobre el trabajo de catorce estudiantes de tercer curso del Grado de Educación Primaria de la Universidad de Granada, España. El estudio sobre las biografías se propone a partir de una demanda de los propios estudiantes en la asignatura de Didáctica de las ciencias sobre la necesidad de conocer el trabajo de mujeres científicas relevantes a lo largo de la historia de la ciencia. La participación en este proyecto fue de carácter voluntario sin ningún tipo de compensación en la calificación de la asignatura, por lo que de un grupo formado por 26 mujeres y 18 hombres participaron 8 mujeres y 6 hombres.

Los estudiantes seleccionaron a criterio individual y totalmente libre la biografía de una mujer relacionada con la ciencia a lo largo de la historia. Tras la lectura individual y análisis (fuera del horario lectivo) extrajeron la información que cada uno por sí mismo consideró relevante sin ningún tipo de condicionamiento o dirección por parte del profesorado en cuanto a la selección de la información. Tras este análisis inicial prepararon una exposición individual sobre su trabajo en la que se les dieron directrices de resaltar aquellos aspectos más significativos para ellos. Antes de iniciar las sesiones de clase un estudiante presentaba su trabajo de forma que las catorce exposiciones se fueron realizando una por semana a lo largo de los cuatro meses que duró la asignatura.

Tras la elección de la biografía y una vez expuesta en el aula, los estudiantes entregaron un informe en el que se les pedía una reflexión sobre el trabajo realizado respondiendo a las siguientes preguntas: A.- ¿Por qué has elegido a esta científica? B.- ¿Qué resaltarías de su biografía? C.-¿Cuáles son las dificultades que destacarías por ser mujeres? Para el análisis de los datos nos hemos valido de un software de análisis cualitativo ATLAS. Ti (software para el análisis visual de datos cualitativos) utilizando un sistema de categorías correspondiente con las preguntas planteadas y subcategorías emergentes del propio análisis de los datos como se puede ver en la tabla 1 . 
Tabla 1. Sistema de categorías emergente. Fuente: elaboración propia.

\begin{tabular}{|l|l|}
\hline $\begin{array}{l}\text { C1. Elección de la } \\
\text { científica }\end{array}$ & \\
\hline $\begin{array}{l}\text { C2. Datos destacables } \\
\text { de la biografía }\end{array}$ & C.2.1. Situación económica \\
& C.2.2. Estado civil \\
& C.2.3. Acceso a estudios \\
& C.2.4. Éxito profesional \\
& C.2.5. Época \\
& C.2.6. Campo científico \\
\hline C3. Características re- & C3.1. Luchadora \\
saltables & C3.2. Superación de dificultades \\
& C3.3. Valentía \\
& C3.4. Pasión \\
& C3.5. Dedicación \\
& C3.6 Buena estudiante \\
& C3.7. Revolucionaria \\
& C3.8. Compromiso político \\
& C3.9. Profesionalidad \\
\hline C4. Dificultades & C4.1. Dificultades sociales y derechos \\
& C4.2. Conciliación \\
& C4.3. Para ejercer la profesión \\
& C4.4. Para ir contracorriente \\
\hline
\end{tabular}

\section{Resultados}

Presentamos los resultados en función del análisis de unidades de información mediante el sistema de categorías anteriormente descrito. Las categorías y las unidades de información están organizadas en relación a las tres preguntas guía que han servido al alumnado para el análisis de las biografías. En este estudio se han analizado un total de catorce trabajos sobre trece biografías de mujeres científicas.

Tabla 2. Listado y datos básicos de las biografías estudiadas. Fuente: elaboración propia.

\begin{tabular}{|c|c|c|c|c|c|}
\hline Nombre & Época & Lugar & $\begin{array}{l}\text { Campo cien- } \\
\text { tífico }\end{array}$ & Trabajos & $\begin{array}{l}\text { Estudiante } \\
\text { M: Mujer } \\
\text { H: Hombre }\end{array}$ \\
\hline *Agnódice 1 & $\begin{array}{ll}\text { Siglo } & \text { IV } \\
\text { a.c. } & \end{array}$ & Grecia & Medicina & $\begin{array}{l}\text { Pionera en Gineco- } \\
\text { logía }\end{array}$ & M1 \\
\hline *Agnódice 2 & $\begin{array}{ll}\text { Siglo } & \text { IV } \\
\text { a.c. } & \end{array}$ & Grecia & Medicina & $\begin{array}{l}\text { Pionera en Gineco- } \\
\text { logía }\end{array}$ & M2 \\
\hline $\begin{array}{l}\text { Caroline Lucretia } \\
\text { Herschel }\end{array}$ & $1750-1848$ & Alemania & Astronomía & $\begin{array}{l}\text { Elaboración de te- } \\
\text { lescopios y descu- } \\
\text { brimientos de come- } \\
\text { tas. }\end{array}$ & M3 \\
\hline Sophie Germain & $1776-1831$ & Francia & Matemática & $\begin{array}{l}\text { Contribuciones a la } \\
\text { teoría de números }\end{array}$ & M4 \\
\hline Mary Somerville & $1780-1872$ & $\begin{array}{l}\text { Reino } \\
\text { Unido }\end{array}$ & $\begin{array}{l}\text { Matemática y } \\
\text { Astrónoma }\end{array}$ & $\begin{array}{l}\text { Contribuciones a la } \\
\text { astronomía y las ma- } \\
\text { temáticas }\end{array}$ & $\mathrm{H} 5$ \\
\hline $\begin{array}{l}\text { Florence } \\
\text { Nightingale }\end{array}$ & $1820-1910$ & Italia & $\begin{array}{l}\text { Enfermería, } \\
\text { Estadística, } \\
\text { Literatura }\end{array}$ & $\begin{array}{l}\text { Profesionalización } \\
\text { de la enfermería }\end{array}$ & M6 \\
\hline $\begin{array}{l}\text { Maria Salomea } \\
\text { Skłodowska-Curie }\end{array}$ & $1867-1934$ & $\begin{array}{l}\text { Polonia- } \\
\text { Francia }\end{array}$ & $\begin{array}{l}\text { Física y Quí- } \\
\text { mica }\end{array}$ & $\begin{array}{l}\text { Premio Nobel de Fí- } \\
\text { sica en 1903, Premio } \\
\text { Nobel de Química } \\
1911\end{array}$ & M7 \\
\hline
\end{tabular}


Tabla 2: Continuación

\begin{tabular}{|c|c|c|c|c|c|}
\hline Nombre & Época & Lugar & $\begin{array}{l}\text { Campo cien- } \\
\text { tífico }\end{array}$ & Trabajos & $\begin{array}{l}\text { Estudiante } \\
\text { M: Mujer } \\
\text { H: Hombre }\end{array}$ \\
\hline Irène Joliot Curie & $1897-1956$ & Francia & $\begin{array}{l}\text { Química y Fí- } \\
\text { sica }\end{array}$ & $\begin{array}{l}\text { Premio Nobel de } \\
\text { Química } 1935\end{array}$ & $\mathrm{H} 8$ \\
\hline Rita Levi-Montalcini & $1909-2012$ & Italia & Neurología & $\begin{array}{l}\text { Premio Nobel de Fi- } \\
\text { siología } 1986\end{array}$ & M9 \\
\hline Chien-Shiung Wu & 1912-1997 & China & Física & $\begin{array}{l}\text { Participó en el desa- } \\
\text { rrollo de la bomba } \\
\text { atómica }\end{array}$ & H10 \\
\hline $\begin{array}{l}\text { Betty Eleanor } \\
\text { Gosset Molesworth } \\
\text { Allen }\end{array}$ & $1913-2002$ & $\begin{array}{l}\text { Nuevazela } \\
\text { nda- } \\
\text { España } \\
\text { (Cádiz) }\end{array}$ & Botánica & $\begin{array}{l}\text { Pteridofitas. Conser- } \\
\text { vación del Parque } \\
\text { Natural de los Al- } \\
\text { cornocales. Cádiz. } \\
\text { España. }\end{array}$ & H11 \\
\hline Rosalind Franklin & $1920-1958$ & $\begin{array}{l}\text { Reino } \\
\text { Unido }\end{array}$ & $\begin{array}{l}\text { Fisicoquímica } \\
\text { y Cristalogra- } \\
\text { fía de rayos X }\end{array}$ & Estructura del ADN & M12 \\
\hline Jane Goodall & 1934 & Tanzania & $\begin{array}{l}\text { Primatología, } \\
\text { Etología y } \\
\text { Antropología }\end{array}$ & $\begin{array}{l}\text { Fundadora del Insti- } \\
\text { tuto Jane Goodall y } \\
\text { el programa Roots } \\
\text { \& Shoots }\end{array}$ & H13 \\
\hline Margarita Salas & 1938 & $\begin{array}{l}\text { España } \\
\text { (Galicia) }\end{array}$ & $\begin{array}{l}\text { Bioquímica y } \\
\text { Biología mo- } \\
\text { lecular }\end{array}$ & $\begin{array}{l}\text { CSIC, Centro de } \\
\text { Biología Molecular } \\
\text { Severo Ochoa }\end{array}$ & H14 \\
\hline
\end{tabular}

\section{A- Elección de la científica.}

La admiración e identificación que el estudiante siente ante la historia de vida de la mujer investigada es el motivo más recurrente para la elección de la científica.

Elegí a Mary Somerville porque me pareció y me parece una mujer digna de admiración, una mujer que pese a todos los problemas que tuvo, siempre siguió adelante, algo que siempre be intentado y con lo que me siento identificado (H5: Mary Somerville).

Destacan otros motivos de la elección precisamente por la invisibilidad de la mujer científica a pesar de las grandes aportaciones a la ciencia con el fin de hacerla visible en las clases de ciencia así como la importancia que ha tenido y tiene para la ciencia los éxitos aportados.

\section{B- Datos destacables de la biografía.}

Los estudiantes mencionan el estado civil de la mujer como característica importante a resaltar en la biografía, presentando al marido/pareja como parte fundamental de su éxito, ya sea en el apoyo recibido o en el desarrollo de trabajo conjunto. Sin embargo no se hace mención a los hijos o familiares a cargo.

Irène se casó en una ceremonia civil con Frédéric, que se convertiría en el compañero con quien compartió su interés en la ciencia, los deportes y sus inquietudes artísticas y bumanistas (H8: Irène Joliot Curie).

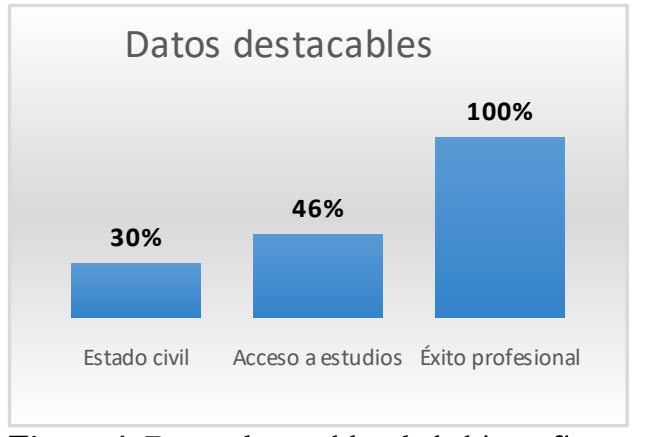

Figura 1. Datos destacables de la biografía. Fuente: elaboración propia 
Otra característica destacada por casi la mitad de los estudiantes hace referencia al acceso a los estudios, ya sea relacionado con las dificultades como con las facilidades encontradas a pesar de la época.

Se ganó su educación de los libros extraídos de la biblioteca (M4: Sophie Germain).

Wu asistió a la primera escuela para niñas de China, que habia fundado y dirigia su padre y, a los nueve años, fue enviada al internado Soochow Girls School (H10: Chien-Shiung Wu).

Es de resaltar que todos los estudiantes destacan el éxito profesional en cuanto a las aportaciones realizadas a la Ciencia así como los premios y reconocimientos por la comunidad científica.

Física y quimica francesa, galardonada con el premio Nobel de Química en 1935 (H8: Irène Joliot Curie).

Fue la primera mujer en ganar un Premio Nobel, en ocupar una cátedra e impartir clases en la Universidad de París y la segunda mujer de la historia en obtener el grado de Doctor en Física (tras Elsa Neumann) (M11: María Salmea Sklodowska).

Ha conseguido ayudar en muchas investigaciones criminales y hoy en día la patente de esta tecnología es una de las más rentables de España. Actualmente es una de las científicas españolas de la época más reconocidas por las sociedades e institutos más prestigiosos a nivel nacional e internacional. Fue nombrada y galardonada con 'Investigadora europea' en 1999 (H14: Margarita Salas).

En algunos casos no sólo destacan sus aportaciones a la ciencia sino también otras cualidades profesionales:

No sólo descubrió ocho cometas, sino que incluso llegó a ser una excelente soprano (M3: Caroline Herschel).

Respecto a la época, predominan las científicas de principios de siglo XX, le siguen científicas del Siglo XIX y mediados XX-XXI. Sólo hay una referencia de la época antigua (S IV a.c.).

En cuanto al campo de investigación al que pertenecen destacan el campo de la física y la química, el campo de la biología y la medicina y el campo de las matemáticas y la astronomía.

En relación a los datos que los estudiantes destacan sobre las características de las mujeres científicas, más de un tercio califican con el atributo de luchadora para definir a la mujer científica en relación con su trabajo y las dificultades encontradas en su época.

Fue una mujer de principios y luchó contra las

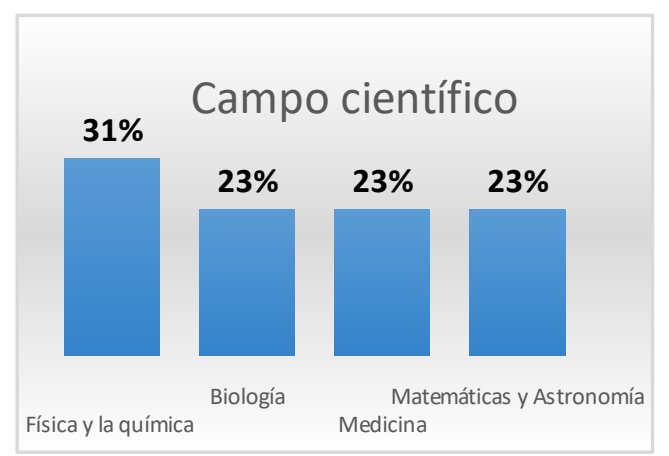

Figura 2. Campo científico. desigualdades (H8: Irène Joliot Curie).

Resaltaría su fuerza para seguir adelante en sus estudios de manera independiente toda su vida (M4: Sopbie Germain).

Respecto a la mención explícita sobre la superación de las dificultades apenas se detecta en una biografía: 
Ella quería ser ginecóloga para ello bizo todo lo que estuvo a su alcance para lograrlo (M2: Agnódice2).

La valentía y pasión son dos características que destacan de las biografías relacionadas directamente con cuestiones de género:

La valentía de Agnódice y de todas aquellas mujeres (M2: Agnódice2).

Gran valentía al desempeñar el trabajo (M1: Agnódice1).

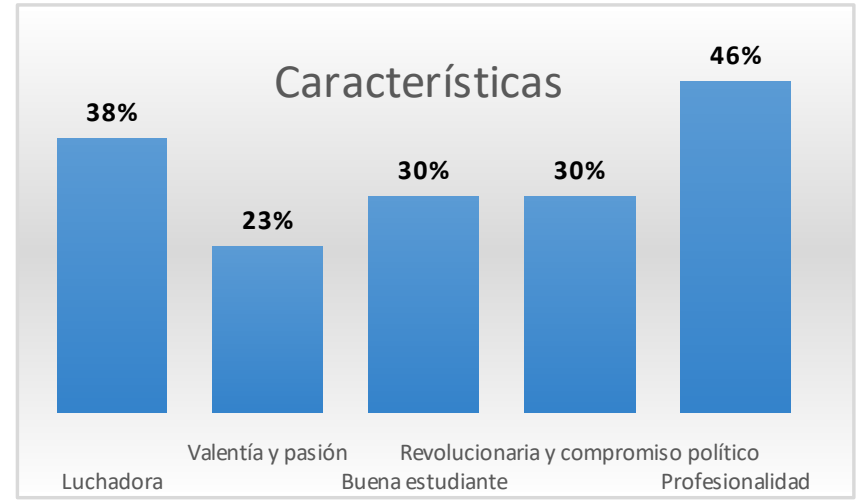

Figura 3. Características.

Fuente: elaboración propia.

Fue una de las mujeres de su tiempo que con más pasión se dedicó al estudio de las matemáticas y al conocimiento de los avances cientificos (H5: Mary Somerville).

Otra cualidad que se resalta en un tercio de los trabajos es la de buena estudiante, destacan-do cualidades de inteligencia y constancia en los estudios.

Se graduó a los diecisiete años con las calificaciones más altas de su clase (H10: Chien-Shiung Wu).

Mostró desde la infancia su inteligencia y su talento excepcional para las matemáticas (H8: Irène Joliot Curie).

Revolucionaria y con compromiso político son características que los estudiantes mencionan sobre las siguientes científicas:

Fue una revolucionaria estudiantil (H10: Chien-Shiung Wu).

Sus padres no querían que estudiara sino que fuera una buena madre y esposa, pero no bizo caso (M9: Rita Levi-Montalcini).

Irène era socialista y demostró en muchas ocasiones su creencia en la igualdad social (H8: Irène Joliot Cu-rie).

Casi la mitad de los estudiantes manifiestan como referente el trabajo y la persona de la mujer científica de estudio.

El caso de Agnódice ha servido de inspiración a muchas mujeres para dedicarse a lo que ellas de verdad quieren (M2: Agnódice).

Participó activamente en la lucha por el desarrollo social e intelectual de las mujeres. Fue miembro del Comité Nacional de la Unión de las Mujeres Francesas y del Consejo para la Paz.Mundial (H8: Irène Joliot Curie).

\section{C- Dificultades que destacan por ser mujeres.}

Respecto a las dificultades que destacan los estudiantes hacen especial referencia a la falta de derechos por ser mujer:

Estudió ciencia en una época en la que las mujeres tenían que pedir "permiso" a sus maridos si querían viajar fuera de España (H14: Margarita Salas).

La pena de muerte era lo que le esperaba por haber ejercido la medicina siendo mujer y fingir que era un hombre (M2: Agnódice2).

Otras dificultades están relacionadas con la cultura patriarcal de la sociedad del momento:

Las dificultades que tuvo para abrirse en el camino de la ciencia siendo mujer, aunque España tuviera en su reloj el siglo XX (H14: Margarita Salas).

A pesar de no contar con el apoyo de su padre ni de la sociedad en su conjunto (M12: Rosalin Franklin). 
Las condiciones de trabajo, que como mujer tuvo que soportar en Cambridge y ciertas palabras despectivas de Watson. Fue entonces la enfermedad y el machismo de los años 50 lo que le privaron del Premio Nobel de Fisiologia y Medicina (M12: Rosalin Franklin)

Por ser mujer, tenía oposición de sus padres (M4: Sophie Germain).

Pero no obtuvo el permiso, y no porque tuvieran algo en particular contra el hospital, sino porque sus padres consideraban que no era un trabajo adecuado para una dama de su posición social (M6: Florence Nightingale).

Otras dificultades están más relacionadas con el ejercicio de la profesión:

Las mujeres lo han tenido siempre muy complicado para poder dedicarse a profesiones que ellas quieran, ya no es que ellas quieran, sino estudiar en si (M2: Agnódice2).

Los estudiantes entienden como dificultad también el ir a contracorriente, es decir, la lucha contra las normas sociales patriarcales de la época:

Las dificultades que ha tenido esta mujer ha sido sobreponerse a los estándares que la sociedad ateniense del siglo IV les imponía a las mujeres (M2: Agnódice2).

Tuvo muchas complicaciones debido a su lucha por la igualdad y la justicia (H8: Irène Joliot Curie).

\section{Discusión y conclusiones}

En cuanto a los motivos sobre la elección de la biografía subrayamos la admiración e identificación que los estudiantes sienten respecto a la mujer científica, convirtiéndose en un icono referente tanto para mujeres como hombres. Es de destacar que el trabajo con modelos científicos femeninos en los contenidos escolares no sólo es fundamental para que las niñas se sientan identificadas a la hora de construir su personalidad, como se menciona en trabajos de Manassero y Vázquez (2013) sino también para los varones, siendo estos modelos referentes claves para aprender otro tipo de ciencia que a su vez moviliza y pone en cuestión el esquema cultural dominante.

Resaltamos el estado civil como uno de los aspectos más mencionados de las biografías. Los estudiantes lo consideran como un aspecto de la vida personal que de alguna forma influye en el desarrollo profesional. Podemos concluir la idea que tienen sobre la íntima relación existente entre desarrollo profesional y vida personal, siendo esta última condicionada por el modelo cultural de la época e influenciado ya sea de manera positiva o negativa en el desarrollo profesional. Esta idea puede llevar a la propuesta de incorporar la importancia de la conciliación de la vida profesional y personal así como la corresponsabilidad en la pareja como contenidos didáctico necesario para potenciar el desarrollo profesional.

El grado de acceso a los estudios es otro de los aspectos más mencionados estableciendo la relación directamente proporcional entre mejor acceso a estudios mejor éxito profesional en la ciencia. Referente que a nivel didáctico tiene su importancia como estímulo para la continuación de los estudios en el campo científico.

Junto a los anteriores aspectos también destacan la época en la que vivió cada científica, su éxito educativo y el éxito profesional. Los estudiantes referencian en todos los trabajos la excelencia profesional, este hecho puede resultar interesante de trabajar a nivel didáctico ya que es muy tentador tener modelos referentes por "la cuenta de resultados" es decir, valorar las biografías por los grandes logros que encajarían en el mismo molde de historias de grandes hombres. Estos resultados nos hace plantear la necesidad de trabajar contenidos didácticos relacionados con la otra ciencia, es decir, visibilizar aquellos espacios excluidos social y 
científicamente donde también se construye conocimiento científico sin estar ni visibilizado en muchos casos ni reconocido su éxito.

Es reseñable que destaquen cualidades de las científicas tales como: luchadora, valiente, apasionada, superación de dificultades, revolucionaria y comprometida políticamente, atributos que se encuentran directamente relacionados con el género. En la línea de Magallón (2016), los procesos de construcción de la ciencia son sociales, por tanto teñidos de valores siendo inevitable que la ciencia refleje los propios valores de las personas que hacen ciencia y del contexto social y cultural donde se produce. Desde un enfoque didáctico, al ser conscientes de que no podemos tener una percepción del mundo que no refleje nuestros intereses y valores "estamos comprometidos a considerar que algunos puntos de vista sobre el mundo -y, por ello, algunos intereses y valores- son mejores que otros" (Putnam 1993, p.209) por tanto, aunque los valores no se pueden eliminar sí se pueden elegir, poniendo en valor la necesidad de contar con los valores femeninos en la construcción de la ciencia.

En relación a las dificultades detectadas relacionadas con el género destacan la falta de derechos de las mujeres y las costumbres machistas de la época. También señalan dificultades relacionadas con la conciliación de la vida familiar y profesional. Desde un enfoque didáctico es necesario poner de manifiesto que aún sigue siendo evidente la existencia de estas dificultades en la mayoría de las culturas, por lo que es fundamental trabajar la perspectiva histórica junto con la realidad actual.

Finalmente, por un lado el trabajo con biografías nos ha permitido aproximarnos a aspectos epistemológicos, históricos e ideológicos de la ciencia desde un enfoque complejo y no neutral proporcionando algunas claves para la formación inicial de maestros y maestras en ciencias y pudiendo servir de base para diseñar propuestas didácticas que trabajen los sesgos de género y potencie cualidades que los propios estudiantes son capaces de desarrollar. Por otro lado, queremos resaltar el valor de transferencia que tiene este trabajo sobre biografías al aula de primaria por ser una propuesta adaptable tanto al contexto del aula como al contexto social y cultural. Propuesta que puede presentar múltiples variables de estudio y permite acercar la historia de la ciencia desde los tiempos más lejanos hasta la ciencia más actual así como el estudio de biografías de mujeres científicas de las propias culturas.

Por último queremos animar a los formadores del profesorado a asumir riesgos y reflexionar en primer lugar, sobre la propia práctica docente desde un enfoque de género para avanzar en las experiencias y prácticas de enseñanza-aprendizaje no sexistas del futuro profesorado así como mejorar su percepción y actitud hacia la asignatura tal y como se sugiere en las conclusiones de estudios similares de Rebollo, García, Vega, Buzón y Barragán (2009). EI papel del futuro profesorado resulta crucial para la superación de los sesgos de género en la enseñanza de la ciencia por lo que la conjunción consciente de diversas estrategias formativas debería llevarnos a promocionar, desde una sociedad más igualitaria, una situación de igualdad real en la enseñanza de las ciencias.

\section{Referencias bibliográficas}

Acevedo Díaz J. A. (2004) Reflexiones sobre las finalidades de la enseñanza de las ciencias: educación científica para la ciudadanía. Revista Eureka sobre Enseñanza y Divulgación de las Ciencias, 1, 3-16.

Álvarez M. y Soneira G. (1991) Enseñanza y aprendizaje de las ciencias experimentales: la coeducación como meta. Memoria final de investigación. Madrid: CIDE. 
Barale P. y María A. (2006) Las mujeres y la ciencia en una sociedad patriarcal en Memorias del I Congreso Iberoamericano de Ciencia, Tecnología, Sociedad e Innovación CTS+ I. Mexico, Organización de Estados Iberoamericanos para la Educación la Ciencia y la Cultura (OEI), en http://www.oei.es/historico/memoriasctsi/index.html

Blanco P. Ruiz-Jarabo C., García de Vinuesa L. y Martín-García M. X. (2004) La violencia de la pareja y la salud de las mujeres. Gaceta Sanitaria 18(4), 182-188.

Eshach, H., y Fried, M. N. (2005) Should science be taught in early childhood? Journal of science education and technology 14(3), 315-336.

Espigado Tocino M., Pascua Sánchez M. y García-Doncel Hernández R. (2004) Mujer y deseo: representaciones y prácticas de vida. Universidad de Cádiz.

García Dauder, S., Pérez Sedeño, E. (2017) Las 'mentiras' científicas sobre las mujeres. Madrid: Catarata.

García C., González A., Aguirre A., Alario T., Brullet C., Carranza E., Gago F., Solsona N., Subirat M., Tomé A., Torres L., Tusón A. y Vega, C. (2002) Mujer y educación: Educar para la igualdad, educar desde la diferencia (Vol. 166). Grao.

García Díaz, J. E. (1995). La transición desde un pensamiento simple hacia un pensamiento complejo en la construcción del conocimiento escolar. Revista Investigación en la Escuela 27, 7-20.

García Nieto M.T. (2013) ¿Son invisibles las mujeres científicas? Estudios sobre el mensaje periodístico. Vol. 19. Núm. Especial abril, 783-792. Madrid: Servicio de Publicaciones de la Universidad Complutense.

Gobierno de España (2015) Decreto 97/2015, de 3 de marzo, por el que se establece la ordenación y el currículo de la educación Primaria en la comunidad Autónoma de Andalucía. España. (Art. 3).

Gómez A., Canales A., Perdomo I., Santana M., Martínez C. y García R. (2008) Sesgos de género en la educación científico-tecnológica: el caso de la Universidad de La Laguna, Arbor, 184(733), 935-947.

Harding S. (1991) Introducción a otra voz. Perspectivas feministas en la vida y en las ciencias sociales. Compilación bibliográfica sobre elementos epistemológicos y metodológicos de la investigación no se-xista. San José, Costa Rica: Instituto Latinoamericano de las Naciones Unidas para la Prevención del Delito y Tratamiento del Delincuente.

Lomas, C. (2002) El sexismo en los libros de texto. En González, A., y Lomas, C. (Coord.), Mujer y educación: educar para la igualdad, educar desde la diferencia (pp. 193-209). Barcelona: Graó.

López-Navajas A. (2014) Análisis de la ausencia de las mujeres en los manuales de la ESO: una genealogía de conocimiento ocultada, Madrid: Ministerio de Educación.

López-Navajas A. y García-Molins A. (2014) El desconocimiento de la tradición literaria femenina y su repercusión en la falta de autoridad social de las mujeres. Quaderns de Filologia Estudis Literaris 17, 27-40.

Maffia, D. (2007) Epistemología feminista: la subversión semiótica de las mujeres en la ciencia. Revista venezolana de estudios de la mujer 12(28), 63-98.

Magallón C. (2004) Pioneras españolas en las ciencias. Las mujeres del Instituto Nacional de Física y Química. Madrid: Consejo Superior de Investigaciones Científicas. 
Magallón C. (2016) Del pasado al futuro: Anotaciones feministas para una ciencia democrática. Cultura de Paz 22(68), 3-9.

Manassero A. y Vázquez A. (2003) Los estudios de género y la enseñanza de las ciencias. Revista de educación 330, 251-280.

Manassero A. y Vázquez A. (2013) Las mujeres científicas: un grupo invisible en los libros de texto. Actualizado en https://www.researchgate.net a partir de la publicación. Re-vista Investigación en la Escuela 50, 31-45.

Marco-Stiefel B. (2004) Alfabetización científica: un puente entre la ciencia escolar y las fronteras científicas. Cultura y Educación 16(3), 273-287.

Muñoz F. y Beatriz J.a (coord.); Álvarez J., Echeverría J., Gimeno J., Martínez J., Nonreb O., Pérez P.,Pujol P., Roig, V., Santos M., Tedesco J.C., Tonucci F. y Zirtae, A. (2012) Pensando en el futuro de la educación: Una nueva escuela para el siglo XXII (Vol. 39), Grao.

Novo M. (2007) Mujer y medio ambiente: los caminos de la visibilidad: utopías, educación y nuevo pa-radigma, (Vol. 5). Madrid: Los Libros de la Catarata.

Pedrinaci E., Caamaño A., Cañal P. y de Pro A. (2013) El desarrollo de la competencia científica. 11 ideas clave, Didácticas Específicas, 245.

Putnam H. (1993) La objetividad y la distinción ciencia-ética. En: Martha C. Nussbaum y Amartya Sen (comp.) La calidad de vida, México, Fondo de Cultura Económica, 193210, p.209.

Rebollo M. Á., García R., Vega L., Buzón O. y Barragán R. (2009) Género y TIC en Educación Superior: recursos virtuales no sexistas para el aprendizaje. Cultura y educación 21(3), 257-274.

Rivero A. y Aguado A.M. (2011) Naturaleza de la ciencia y construcción del conocimiento científico: La naturaleza de la ciencia como objetivo de enseñanza, en Pedro Cañal (coord.), Biología y geología: complementos de formación disciplinar (pp. 9-30).

Rivero A., del Pozo R. M., Solís E., Azcárate P. y Porlán R. (2017) Cambio del conocimiento sobre la enseñanza de las ciencias de futuros maestros. Enseñanza de las ciencias: revista de investigación y experiencias didácticas 35(1), 29-52.

Rodríguez Martínez C. (2004) La ausencia de las mujeres en los contenidos escolares. Madrid: Miño y Dávila Editores.

Smail B., Whyte J., Kelly A. (1982) Girls into science and technology: The first two years. School Science Review 63(225), 620-630.

Urteaga E. (2010) Ciencia y género. Clepsydra, 121-132.

Vega Pindado P., Herrero Y., Torres Benavides M., Ramdán Ahmed Z. y Puleo A. (2010) Mujeres y medio ambiente: admiraciones e interrogantes. Madrid: ACSUR Las Segovias.

Vázquez A., Acevedo J., Manassero M. y Acevedo P (2001) Cuatro paradigmas básicos sobre la naturaleza de la ciencia. Argumentos de Razón Técnica 4, 135-176. 